\title{
THE EXISTENCE RESULTS OF COUPLED QUASI-SOLUTIONS FOR A CLASS OF OPERATOR EQUATIONS
}

\author{
Yeol Je Cho, Guang He, and Nan-Jing Huang
}

\begin{abstract}
In this paper, by using the semi-order method, two new existence theorems of coupled quasi-solutions for a class of nonlinear operator equations in Banach spaces are proved under some suitable conditions.
\end{abstract}

\section{Introduction}

It is well known that the nonlinear operator theory plays an important role in nonlinear functional analysis, nonlinear programmings, nonlinear differential equations, nonlinear variational inequalities and complementarity problems (see, for example, $[2,1,6]$ and the references therein).

In 1987, Guo and Lakshmikantham [7] studied the following nonlinear operator equation:

$$
x=A(x, x),
$$

where $A$ is a mixed monotone operator in Banach spaces. They obtained some existence results of coupled solution for the nonlinear operator equation (1.1). A generalization of (1.1) involving set-valued operators was introduced and studied by Huang, Tang, and Liu [9].

Recently, Feng and Liu [5] considered the following operator equation:

$$
N x=A x
$$

in complete metric spaces and Banach spaces, respectively. They obtained some existence results of solution for the equation (1.2) by using the technique of partial order theory.

Received July 3, 2008; Revised February 26, 2009.

2000 Mathematics Subject Classification. 47H10, 54H25.

Key words and phrases. cone, partial order, mixed monotone operator, relatively weakly compact set, coupled quasi-solutions.

This work was supported by the Korea Research Foundation Grant funded by the Korean Government (MOEHRD) (KRF-2007-313-C00040), the National Natural Science Foundation of China (10671135, 70831005) and the Specialized Research Fund for the Doctoral Program of Higher Education (20060610005). 
Very recently, He et al. [8] introduced and studied the following nonlinear operator equation:

$$
N x=A(x, x)
$$

in Banach spaces. By using the concept of $\phi$ concave- $(-\psi)$ convex operator introduced by $\mathrm{Xu}$ and Jia [12], they obtained some new solvability results for the nonlinear operator equation (1.3).

On the other hand, by using the semi-order method, Duan and Li [4] studied the existence of coupled minimal-maximal quasi-solutions for the nonlinear operator equation (1.1) in Banach spaces and proved some new existence results.

Motivated and inspired by the works mentioned above, in this paper, we further study the solvability of coupled quasi-solutions for the nonlinear operator equation (1.3) by using the semi-order method. Under some suitable conditions, we prove two new existence theorems of coupled quasi-solutions for the nonlinear operator equation (1.3) in Banach spaces.

\section{Preliminaries}

Let $E$ be a real Banach space. A nonempty convex closed set $P$ is called a cone if it satisfies the following conditions:

(i) $x \in P$ and $\lambda \geq 0$ imply that $\lambda x \in P$;

(ii) $x \in P$ and $-x \in P$ imply that $x=\theta$, where $\theta$ denotes the zero element of $E$.

Let $(E, \leq)$ be a partial ordering space induced by the cone $P$ of $E$, i.e., for any $x, y \in E, x \leq y$ if and only if $y-x \in P$.

Now, we define a norm in $E \times E$ as follows:

$$
\|(x, y)\|_{E \times E}=\max \{\|x\|,\|y\|\}, \quad \forall x, y \in E .
$$

Obviously, $E \times E$ is a Banach space with the norm $\|(\cdot, \cdot)\|$.

Let

$$
P_{1}=\{(x, y) \in E \times E: x \geq \theta, y \leq \theta\},
$$

where $\theta$ denotes the zero element of $E$. It is easy to see that $P_{1}$ is a cone in $E \times E$ and $P_{1}$ defines a partial order in $E \times E$ as follows:

$$
(x, y) \leq(u, v) \text { if and only if } x \leq u \text { and } y \geq v \text {. }
$$

Definition 2.1. Let $D$ be a nonempty subset of $E$. An operator $A: D \times D \rightarrow E$ is said to be mixed monotone if, for any $x_{i}, y_{i} \in D$ with $i=1,2, x_{1} \leq x_{2}$ and $y_{2} \leq y_{1}$ imply that

$$
A\left(x_{1}, y_{1}\right) \leq A\left(x_{2}, y_{2}\right) .
$$

Definition 2.2. A point $\left(x^{*}, y^{*}\right) \in D \times D$ is said to be:

(i) a coupled solution of the nonlinear operator equation (1.3) if

$$
N x^{*}=A\left(x^{*}, y^{*}\right), \quad N y^{*}=A\left(y^{*}, x^{*}\right) ;
$$


(ii) a coupled minimal-maximal solution of the nonlinear operator equation (1.3) if $\left(x^{*}, y^{*}\right)$ is a coupled solution of the nonlinear operator equation (1.3) such that, for any coupled solution $\left(u^{*}, v^{*}\right)$ of $(1.3), x^{*} \leq u^{*}$ and $y^{*} \geq v^{*}$.

Definition 2.3. Let $L(E)$ be the space of all linear operators on $E$. $T \in L(E)$ is said to be a linear positive operator if $x \geq \theta$ implies $T x \geq \theta$.

Definition 2.4. Let $x_{0}, y_{0} \in E$ with $x_{0}<y_{0}$. The set defined by

$$
\left[x_{0}, y_{0}\right]=\left\{z \mid x_{0} \leq z \leq y_{0}\right\}
$$

is called an ordered interval in $E$.

We assume that the following conditions are satisfied:

$\left(H_{1}\right)$ There is $\lambda \in(0,1]$ such that $(\lambda I+T)^{-1} \in L(E)$ exists and

$$
(\lambda I+T)^{-1} x \geq \theta \Longrightarrow x \in P
$$

$\left(H_{2}\right) T: E \rightarrow E$ is a bounded linear positive operator.

For any subset $D$ of $E$, throughout this paper, we denote the weak closure of $D$, the closed convex hull of $D$ under the norm $\|(\cdot, \cdot)\|_{E \times E}$, and the complement of $D$ by $\bar{D}^{w}, \overline{c o}(D)$, and $C D$, respectively.

Lemma 2.1. If $T \in L(E)$ and there exists $\lambda \in(0,1]$ with $(\lambda I+T)^{-1} \in L(E)$, then

$$
(\lambda I+T)^{-1}[\lambda A(x, y)+T u]=u \Longleftrightarrow A(x, y)=u
$$

and

$$
(\lambda I+T)^{-1}[\lambda A(y, x)+T v]=v \Longleftrightarrow A(y, x)=v,
$$

where $u=N x$ and $v=N y$.

Proof. Obviously,

if and only if

$$
(\lambda I+T)^{-1}[\lambda A(x, y)+T u]=u
$$

$$
\lambda A(x, y)+T u=(\lambda I+T) u
$$

and $\lambda A(x, y)=\lambda u$ if and only if $A(x, y)=u$. It follows that (2.2) is true. Similarly, we can show that (2.3) holds. This completes the proof.

Lemma $2.2([3])$. If $T \in L(E)$ satisfies the condition $\left(H_{1}\right)$ for some $\lambda \in(0,1]$, then $(\lambda I+T)^{-1}$ is a positive linear operator.

Lemma 2.3. Let $G: D \times D \rightarrow E$ be a mixed monotone operator and $N$ be a nonlinear operator. Let

$$
H(x, y) \triangleq(G(x, y), G(y, x)), \quad B(x, y) \triangleq(N x, N y), \quad \forall(x, y) \in D \times D .
$$

Then the following conclusions hold:

(1) $H$ is an increasing operator on the partial ordering deduced by $P_{1}$; 
(2) $H(x, y)=B(x, y)$ has a solution $\left(x^{*}, y^{*}\right)$ if and only if $\left(x^{*}, y^{*}\right)$ is a coupled solution of $N x=G(x, x)$;

(3) A minimal solution of $H(x, y)=B(x, y)$ is a coupled minimal-maximal solution of $N x=G(x, x)$.

Proof. By [16], we know that (1) holds.

Now, we show that $(2)$ is true. In fact, $\left(x^{*}, y^{*}\right)$ is a solution of $H(x, y)=$ $B(x, y)$ if and only if $\left(x^{*}, y^{*}\right)$ is a solution of $(N x, N y)=(G(x, y), G(y, x))$, i.e.,

$$
N x^{*}=G\left(x^{*}, y^{*}\right), \quad N y^{*}=G\left(y^{*}, x^{*}\right) .
$$

Thus $H(x, y)=B(x, y)$ has a solution $\left(x^{*}, y^{*}\right)$ if and only if $\left(x^{*}, y^{*}\right)$ is a coupled solution of $N x=G(x, x)$.

Next, we prove that (3) holds. Suppose that $\left(u^{*}, v^{*}\right)$ is a minimal solution of $H(x, y)=B(x, y)$. For any solution $(u, v)$ of $H(x, y)=B(x, y)$, we know that $\left(u^{*}, v^{*}\right) \leq(u, v)$. It follows from (2.1) that $u^{*} \leq u$ and $v \leq v^{*}$. By $(2)$ and Definition 2.2 , it is easy to see that $\left(u^{*}, v^{*}\right)$ is a coupled minimal-maximal solution of $N x=G(x, x)$. This completes the proof.

Lemma 2.4 ([10]). Let $(E, P)$ be a partial ordering Banach space, $D$ be a nonempty subset of $E$, and $y \in E$. If $z \leq y$ (resp., $y \leq z$ ) for all $z \in D$, then $z \leq y$ (resp., $y \leq z)$ for all $z \in \overline{c o}(D)$.

\section{Main results}

Theorem 3.1. Let $E$ be a real Banach space and $P$ a cone of $E$. Let $D_{0}=$ $\left[u_{0}, v_{0}\right]$ be an ordered interval in $E$ and $N$ be an increasing operator with $N\left(D_{0}\right)=D_{0}$. Suppose that an operator

$$
A: D \triangleq\left[\left(u_{0}, v_{0}\right),\left(v_{0}, u_{0}\right)\right] \rightarrow E
$$

satisfies the conditions $\left(H_{1}\right)$ and $\left(H_{2}\right)$. If

(i) $N u_{0} \leq A\left(u_{0}, v_{0}\right)$ and $A\left(v_{0}, u_{0}\right) \leq N v_{0}$;

(ii) For any $u_{0} \leq x_{1} \leq x_{2} \leq v_{0}$ and $u_{0} \leq y_{1} \leq y_{2} \leq v_{0}$, we have

$$
A\left(x_{2}, y_{1}\right)-A\left(x_{1}, y_{2}\right) \geq-T\left(u_{2}, u_{1}\right),
$$

where $u_{1}=N x_{1}$ and $u_{2}=N x_{2}$;

(iii) For any $x_{1}, x_{2} \in D_{0}, N x_{1} \leq N x_{2}$ implies $x_{1} \leq x_{2}$;

(iv) Any totally ordered subset in $G(D)$ is relatively compact with weak topology, where

$$
G(x, y) \triangleq(\lambda I+T)^{-1}[\lambda A(x, y)+T u]
$$

and $u=N x$ for all $(x, y) \in D$.

Then the nonlinear operator equation (1.3) has a coupled quasi-solution $\left(x^{*}, y^{*}\right)$ $\in D$. 
Proof. It follows from the condition $\left(H_{1}\right)$ and Lemma 2.2 that $(\lambda I+T)^{-1}$ is a positive operator. By the assumption $\left(H_{2}\right)$ and the conditions (i) and (ii), we have

(I) $G: D \rightarrow\left[u_{0}, v_{0}\right]$ is a mixed monotone operator;

(II) $N u_{0} \leq G\left(u_{0}, v_{0}\right), G\left(v_{0}, u_{0}\right) \leq N v_{0}$.

Now we show that the following nonlinear operator equation

$$
B(x, y)=H(x, y)
$$

has a solution in $D$, where

$$
H(x, y) \triangleq(G(x, y), G(y, x)), \quad B(x, y) \triangleq(N(x), N(y)) .
$$

From (I) and Lemma 2.3, it is easy to see that $H$ is an increasing operator. Let

$$
M_{1}=\{(x, y) \in D:(N x, N y) \leq H(x, y)\}, \quad M_{2}=\left\{(y, x):(x, y) \in M_{1}\right\} .
$$

By (II), we have $\left(u_{0}, v_{0}\right) \in M_{1}$ and so $M_{1}$ is nonempty. Suppose that $K_{1}$ is a total ordering subset of $M_{1}$. Then $K_{2}=\left\{(y, x) \mid(x, y) \in K_{1}\right\}$ is a total ordering subset of $M_{2}$. For any fixed $w \in D_{0}$, let

$$
R_{1}(w)=\left\{z \in D_{0}: w \leq z\right\}, \quad R_{2}(w)=\left\{z \in D_{0}: z \leq w\right\} .
$$

Since $P$ is closed and convex, we know that $R_{1}(w)$ and $R_{2}(w)$ are both closed and convex. For any $q_{1} \in G\left(K_{1}\right)$ and $q_{2} \in G\left(K_{2}\right)$, let

$$
S_{1}\left(q_{1}\right)=\overline{c o}\left(G\left(K_{1}\right)\right) \bigcap R_{1}\left(q_{1}\right), \quad S_{2}\left(q_{2}\right)=\overline{c o}\left(G\left(K_{2}\right)\right) \bigcap R_{2}\left(q_{2}\right) .
$$

Since $R_{1}\left(q_{1}\right)$ and $R_{2}\left(q_{2}\right)$ are convex and closed, it is easy to see that $S_{1}\left(q_{1}\right)$ and $S_{2}\left(q_{2}\right)$ are both convex and closed. Now the mixed monotonicity of $G$ implies that $G\left(K_{i}\right)(i=1,2)$ are total ordering subsets of $G(D)$. From the condition (iv), we know that ${\overline{G\left(K_{i}\right)}}^{w}(i=1,2)$ are weakly compact sets in $G(D)$. It follows from Krein-Smulian Theorem (see, for example, [2] or [13]) that $\overline{c o}\left(\overline{G\left(K_{i}\right)}\right)^{w}(i=1,2)$ are also weakly compact. Since

$$
\overline{c o}\left(G\left(K_{i}\right)\right) \subset \overline{c o}\left(\overline{G\left(K_{i}\right)}\right)^{w}(i=1,2),
$$

we know that $\overline{c o}\left(G\left(K_{i}\right)\right)(i=1,2)$ are weakly compact.

For any $q_{i} \in G\left(K_{i}\right)(i=1,2)$, we have $q_{i} \in S_{i}\left(q_{i}\right)$ and so $S_{i}\left(q_{i}\right)(i=1,2)$ are nonempty. For any $q_{1}^{\prime}, q_{2}^{\prime}, \ldots, q_{n}^{\prime} \in G\left(K_{1}\right)$ and $q_{1}^{\prime \prime}, q_{2}^{\prime \prime}, \ldots, q_{n}^{\prime \prime} \in G\left(K_{2}\right)$, without loss of generality, we can suppose that

$$
q_{1}^{\prime} \leq q_{2}^{\prime} \leq \cdots \leq q_{n}^{\prime} \quad \text { and } \quad q_{1}^{\prime \prime} \leq q_{2}^{\prime \prime} \leq \cdots \leq q_{n}^{\prime \prime} .
$$

Thus we have

$$
S_{1}\left(q_{1}^{\prime}\right) \supset S_{1}\left(q_{2}^{\prime}\right) \supset \cdots \supset S_{1}\left(q_{n}^{\prime}\right)
$$

and

It follows that

$$
S_{2}\left(q_{1}^{\prime \prime}\right) \subset S_{2}\left(q_{2}^{\prime \prime}\right) \subset \cdots \subset S_{2}\left(q_{n}^{\prime \prime}\right)
$$

$$
\emptyset \neq S_{1}\left(q_{n}^{\prime}\right) \subset \bigcap_{i=1}^{n} S_{1}\left(q_{i}^{\prime}\right), \quad S_{2}\left(q_{1}^{\prime \prime}\right) \subset \bigcap_{i=1}^{n} S_{2}\left(q_{i}^{\prime \prime}\right) \neq \emptyset .
$$


Now, we prove that $\bigcap_{q_{i} \in G\left(K_{i}\right)} S_{i}\left(q_{i}\right)(i=1,2)$ are nonempty. In fact, suppose that $\bigcap_{q_{i} \in G\left(K_{i}\right)} S_{i}\left(q_{i}\right)=\emptyset$ for $i=1,2$. Then we have

$$
\overline{c o}\left(G\left(K_{i}\right)\right) \subset \bigcup_{q_{i} \in G\left(K_{i}\right)} C S_{i}\left(q_{i}\right), \quad i=1,2 .
$$

It follows from (3.3) that $\left\{C S_{i}\left(q_{i}\right): q_{i} \in G\left(K_{i}\right)\right\}$ is an open covering of $\overline{c o}\left(G\left(K_{i}\right)\right)$ under weakly topology for $i=1,2$. Since $\overline{c o}\left(G\left(K_{i}\right)\right)$ is weakly compact, $\overline{c o}\left(G\left(K_{i}\right)\right)$ has a finite sub-covering for $i=1,2$. Thus there exist $q_{1}^{\prime}, q_{2}^{\prime}, \ldots, q_{m}^{\prime} \in G\left(K_{1}\right)$ and $q_{1}^{\prime \prime}, q_{2}^{\prime \prime}, \ldots, q_{l}^{\prime \prime} \in G\left(K_{2}\right)$ such that

$$
\overline{c o}\left(G\left(K_{1}\right)\right) \subset \bigcup_{i=1}^{m} C S_{1}\left(q_{i}^{\prime}\right), \quad \overline{c o}\left(G\left(K_{2}\right)\right) \subset \bigcup_{i=1}^{l} C S_{2}\left(q_{i}^{\prime \prime}\right) .
$$

Noticing

$$
S_{1}\left(q_{i}^{\prime}\right) \subset \overline{c o}\left(G\left(K_{1}\right)\right), \quad \forall i=1,2, \ldots, m,
$$

and

$$
S_{2}\left(q_{i}^{\prime \prime}\right) \subset \overline{c o}\left(G\left(K_{2}\right)\right), \quad \forall i=1,2, \ldots, l,
$$

it follows from (3.4) that

$$
\bigcap_{i=1}^{m} S_{1}\left(q_{i}^{\prime}\right) \subset \overline{c o}\left(G\left(K_{1}\right)\right) \subset \bigcup_{i=1}^{m} C S_{1}\left(q_{i}^{\prime}\right), \quad \bigcap_{i=1}^{l} S_{2}\left(q_{i}^{\prime \prime}\right) \subset \overline{c o}\left(G\left(K_{2}\right)\right) \subset \bigcup_{i=1}^{l} C S_{2}\left(q_{i}^{\prime \prime}\right)
$$

and so

$$
\bigcap_{i=1}^{m} S_{1}\left(q_{i}^{\prime}\right)=\emptyset, \quad \bigcap_{i=1}^{l} S_{2}\left(q_{i}^{\prime \prime}\right)=\emptyset
$$

which contradicts (3.2). Therefore, $\bigcap_{q_{i} \in G\left(K_{i}\right)} S_{i}\left(q_{i}\right)$ is nonempty and there exists $q_{i}^{*} \in \bigcap_{q_{i} \in G\left(K_{i}\right)} S_{i}\left(q_{i}\right)$ such that $q_{i}^{*} \in S_{i}\left(q_{i}\right)$ for all $q_{i} \in G\left(K_{i}\right)$ with $i=1,2$. Thus $q_{i}^{*} \in R_{i}\left(q_{i}\right)$ for all $q_{i} \in G\left(K_{i}\right)$. By the construction of $R_{i}\left(q_{i}\right)$, we have

$$
q_{1} \leq q_{1}^{*}, \quad q_{2} \geq q_{2}^{*} .
$$

Since $N\left(D_{0}\right)=D_{0}$, we know that there exist $w_{1}, w_{2} \in D_{0}$ such that

$$
N w_{1}=q_{1}^{*}, \quad N w_{2}=q_{2}^{*} .
$$

Now, for any $(x, y) \in K_{1}$, we have $(y, x) \in K_{2}$. It follows from (3.5) that

$$
G(x, y) \leq q_{1}^{*}=N w_{1}, \quad N w_{2}=q_{2}^{*} \leq G(y, x) .
$$

Since $K_{1} \subset M_{1}$,

$$
(N x, N y) \leq H(x, y)=(G(x, y), G(y, x))
$$

and so

$$
N x \leq G(x, y), \quad G(y, x) \leq N y
$$

Thus it follows that

$$
N x \leq N w_{1}, \quad N w_{2} \leq N y
$$


From the condition (3), we have

$$
x \leq w_{1}, \quad w_{2} \leq y .
$$

Now, (2.1) implies that

$$
(x, y) \leq\left(w_{1}, w_{2}\right)
$$

and so $\left(w_{1}, w_{2}\right)$ is an upper bound of $K_{1}$.

Next, we show that $\left(w_{1}, w_{2}\right) \in M_{1}$, that is,

$$
B\left(w_{1}, w_{2}\right)=\left(N w_{1}, N w_{2}\right) \leq H\left(\left(w_{1}, w_{2}\right)\right) .
$$

In fact, for any $(x, y) \in K_{1}$, we have $(y, x) \in K_{2}$ and so

$$
(x, y) \leq\left(w_{1}, w_{2}\right), \quad(y, x) \geq\left(w_{2}, w_{1}\right) .
$$

Now, the mixed monotonicity of $G$ implies that

$$
G(x, y) \leq G\left(w_{1}, w_{2}\right), \quad G(y, x) \geq G\left(w_{2}, w_{1}\right) .
$$

It follows from Lemma 2.4 that, for $N w_{i}=q_{i}^{*} \in R_{i}\left(q_{i}\right) \subset \overline{c o}\left(G\left(K_{i}\right)\right)(i=1,2)$,

$$
N w_{1} \leq G\left(w_{1}, w_{2}\right), \quad G\left(w_{2}, w_{1}\right) \leq N w_{2}
$$

and so

$$
\left(N w_{1}, N w_{2}\right) \leq\left(G\left(w_{1}, w_{2}\right), \quad G\left(w_{2}, w_{1}\right)\right)=H\left(w_{1}, w_{2}\right) .
$$

Now, (3.7) shows that $\left(w_{1}, w_{2}\right) \in M_{1}$. It follows from Zorn's lemma that $M_{1}$ contains a maximal element $\left(x^{*}, y^{*}\right)$.

Finally, we prove that $\left(x^{*}, y^{*}\right)$ is a solution of the nonlinear operator equation (3.1). We first show the following conclusion holds:

$$
B\left(x_{1}, y_{1}\right) \leq B\left(x_{2}, y_{2}\right),\left(x_{i}, y_{i}\right) \in D(i=1,2) \Longrightarrow\left(x_{1}, y_{1}\right) \leq\left(x_{2}, y_{2}\right) \text {. }
$$

In fact, it follows from the definition of $B$ that

$$
B\left(x_{1}, y_{1}\right)=\left(N x_{1}, N y_{1}\right) \leq\left(N x_{2}, N y_{2}\right)=B\left(x_{2}, y_{2}\right)
$$

and so

$$
N x_{1} \leq N x_{2}, \quad N y_{2} \leq N y_{1} .
$$

By the condition (iii), we have $x_{1} \leq x_{2}$ and $y_{2} \leq y_{1}$ and so $\left(x_{1}, y_{1}\right) \leq\left(x_{2}, y_{2}\right)$. Since $\left(x^{*}, y^{*}\right) \in M_{1}$,

$$
B\left(x^{*}, y^{*}\right) \leq H\left(x^{*}, y^{*}\right)=B\left(B^{-1} H\left(x^{*}, y^{*}\right)\right)
$$

and so (3.8) implies that

$$
\left(x^{*}, y^{*}\right) \leq B^{-1} H\left(x^{*}, y^{*}\right) .
$$

By the monotonicity of $H$, we have

$$
B\left(B^{-1} H\left(x^{*}, y^{*}\right)\right)=H\left(x^{*}, y^{*}\right) \leq H\left(B^{-1} H\left(x^{*}, y^{*}\right)\right)
$$

and so, $B^{-1} H\left(x^{*}, y^{*}\right) \in M_{1}$. We next show that $B$ is an increasing operator. If $\left(x_{1}, y_{1}\right) \leq\left(x_{2}, y_{2}\right)$, then $x_{1} \leq x_{2}$ and $y_{2} \leq y_{1}$. By the monotonicity of $N$, we have

$$
N x_{1} \leq N x_{2}, \quad N y_{2} \leq N y_{1}
$$


and so

$$
B\left(x_{1}, y_{1}\right)=\left(N x_{1}, N y_{1}\right) \leq\left(N x_{2}, N y_{2}\right)=B\left(x_{2}, y_{2}\right)
$$

Thus $B$ is an increasing operator. Since $\left(x^{*}, y^{*}\right)$ is a maximal element of $M_{1}$ and $B$ is increasing, we have

$$
B\left(B^{-1} H\left(x^{*}, y^{*}\right)\right)=H\left(x^{*}, y^{*}\right) \leq B\left(x^{*}, y^{*}\right)
$$

and so $H\left(x^{*}, y^{*}\right)=B\left(x^{*}, y^{*}\right)$, that is,

$$
N x^{*}=G\left(x^{*}, y^{*}\right), \quad N y^{*}=G\left(y^{*}, x^{*}\right) .
$$

It follows from Lemma 2.1 that

$$
N x^{*}=A\left(x^{*}, y^{*}\right), \quad N y^{*}=A\left(y^{*}, x^{*}\right)
$$

and so $\left(x^{*}, y^{*}\right)$ is a coupled quasi-solution of the equation (1.3). This completes the proof.

Theorem 3.2. Suppose that all the conditions of Theorem 3.1 are satisfied. Then the nonlinear operator equation (1.3) has a coupled minimal and maximal quasi-solution $\left(x^{*}, y^{*}\right) \in D$.

Proof. Let $F(H)=\{(x, y) \in D: H(x, y)=B(x, y)\}$. Then Theorem 3.1 implies that $F(H)$ is nonempty. Let

$$
S \triangleq\{[(u, v),(v, u)]: B(u, v) \leq H(u, v),(u, v) \in D, F(H) \subset[(u, v),(v, u)]\},
$$

where $[(u, v),(v, u)]$ is an ordered interval in $E \times E$. It is easy to see that $D \in S$ and so $S \neq \emptyset$. We define the ordering " $\leq$ " in $S$ as follows:

$$
I_{1}, I_{2} \in S, \quad I_{1} \leq I_{2} \Longleftrightarrow I_{1} \subset I_{2} .
$$

Now, we show that $S$ has a minimal element. Suppose that

$$
\Gamma=\left\{\left[\left(u_{\alpha}, v_{\alpha}\right),\left(v_{\alpha}, u_{\alpha}\right)\right]: \alpha \in \Lambda\right\}
$$

is a total ordering subset of $S$, where $\Lambda$ is an index set. Let

$$
R_{1}=\left\{\left(u_{\alpha}, v_{\alpha}\right): \alpha \in \Lambda\right\}, R_{2}=\left\{\left(v_{\alpha}, u_{\alpha}\right): \alpha \in \Lambda\right\} .
$$

Then $R_{1}$ and $R_{2}$ are total ordering subsets of $D$. It follows from the mixed monotonicity of $G$ that $G\left(R_{i}\right)(i=1,2)$ are total ordering subsets of $E$.

Let $K_{1}=R_{1}$ and $K_{2}=R_{2}$ be the same as in the proof of Theorem 3.1. Then, by the similar proofs for (3.6) and (3.7), we know that there exist $\overline{q_{i}} \in$ $\overline{c o}\left(G\left(R_{i}\right)\right)(i=1,2)$ with $N \overline{w_{i}}=\overline{q_{i}}$ such that

$$
\left(u_{\alpha}, v_{\alpha}\right) \leq\left(\overline{w_{1}}, \overline{w_{2}}\right), \quad \forall \alpha \in \Lambda,
$$

and

$$
B\left(\overline{w_{1}}, \overline{w_{2}}\right)=\left(N \overline{w_{1}}, N \overline{w_{2}}\right) \leq H\left(\overline{w_{1}}, \overline{w_{2}}\right) .
$$

Let $(x, y) \in F(H)$. For any given $\left(u_{\alpha}, v_{\alpha}\right) \in R_{1}$, we have $\left(v_{\alpha}, u_{\alpha}\right) \in R_{2}$. It follows from the definition of $S$ that

$$
\left(u_{\alpha}, v_{\alpha}\right) \leq(x, y) \leq\left(v_{\alpha}, u_{\alpha}\right) .
$$


Now, the mixed monotonicity of $G$ implies that

$$
G\left(u_{\alpha}, v_{\alpha}\right) \leq G(x, y) \leq G\left(v_{\alpha}, u_{\alpha}\right) .
$$

Since $\overline{q_{i}} \in \overline{c o}\left(G\left(R_{i}\right)\right)$, Lemma 2.4 implies that

$$
N \overline{w_{1}} \leq G(x, y) \leq N \overline{w_{2}} .
$$

Let $(x, y) \in F(H)$. For any given $\left(v_{\alpha}, u_{\alpha}\right) \in R_{2}$, it is easy to see that $\left(u_{\alpha}, v_{\alpha}\right) \in$ $R_{1}$. Similarly to the proof of (3.12), we have

$$
N \overline{w_{1}} \leq G(x, y) \leq N \overline{w_{2}} .
$$

Thus it follows that

$$
\left(N \overline{w_{1}}, N \overline{w_{2}}\right) \leq(G(x, y), G(y, x))=H(x, y)=(N x, N y)
$$

and

$$
\left(N \overline{w_{2}}, N \overline{w_{1}}\right) \geq(G(x, y), G(y, x))=H(x, y)=(N x, N y) .
$$

By the condition (3),

$$
\left(\overline{w_{1}}, \overline{w_{2}}\right) \leq(x, y) \leq\left(\overline{w_{2}}, \overline{w_{1}}\right), \quad \forall(x, y) \in F(H) .
$$

Let

$$
I=\left[\left(\overline{w_{1}}, \overline{w_{2}}\right),\left(\overline{w_{2}}, \overline{w_{1}}\right)\right] .
$$

Then it follows from (3.11) and (3.13) that $I \in S$.

Now, we prove that $I$ is a lower bound of $\Gamma$. In fact, for any $\alpha \in \Lambda,(3.10)$ implies that

which show that

$$
\left(u_{\alpha}, v_{\alpha}\right) \leq\left(\overline{w_{1}}, \overline{w_{2}}\right), \quad\left(v_{\alpha}, u_{\alpha}\right) \geq\left(\overline{w_{2}}, \overline{w_{1}}\right)
$$

$$
I \subset\left[\left(u_{\alpha}, v_{\alpha}\right),\left(v_{\alpha}, u_{\alpha}\right)\right]
$$

and so $I$ is a lower bound of $\Gamma$ in $S$. It follows from Zorn's lemma that $S$ contains a minimal element $I^{*}$. Let $I^{*}=\left[\left(x^{*}, y^{*}\right),\left(y^{*}, x^{*}\right)\right]$. Then

$$
B\left(x^{*}, y^{*}\right) \leq H\left(x^{*}, y^{*}\right)=B\left(B^{-1} H\left(x^{*}, y^{*}\right)\right) .
$$

From (3.8), we have $\left(x^{*}, y^{*}\right) \leq B^{-1} H\left(x^{*}, y^{*}\right)$. By the monotonicity of $H$,

$$
B\left(B^{-1} H\left(x^{*}, y^{*}\right)\right)=H\left(x^{*}, y^{*}\right) \leq H\left(B^{-1} H\left(x^{*}, y^{*}\right)\right) .
$$

For any $(x, y) \in F(H)$, the monotonicity of $H$ and the definition of $S$ show that

$$
H\left(x^{*}, y^{*}\right) \leq H(x, y)=B(x, y) \leq H\left(y^{*}, x^{*}\right)
$$

and so

$$
B\left(B^{-1} H\left(x^{*}, y^{*}\right)\right) \leq B\left(B^{-1} H(x, y)\right)=B(x, y) \leq B\left(B^{-1} H\left(y^{*}, x^{*}\right)\right) .
$$

It follows from (3.8) that

$$
B^{-1} H\left(x^{*}, y^{*}\right) \leq(x, y) \leq B^{-1} H\left(y^{*}, x^{*}\right)
$$

and so $\left[B^{-1} H\left(x^{*}, y^{*}\right), B^{-1} H\left(y^{*}, x^{*}\right)\right] \in S$. Therefore, we have

$$
\left(x^{*}, y^{*}\right) \leq B^{-1} H\left(x^{*}, y^{*}\right), \quad\left(y^{*}, x^{*}\right) \geq B^{-1} H\left(y^{*}, x^{*}\right) .
$$


It follows from (3.9) that

$$
I^{*}=\left[\left(x^{*}, y^{*}\right),\left(y^{*}, x^{*}\right)\right] \geq\left[B^{-1} H\left(x^{*}, y^{*}\right), B^{-1} H\left(y^{*}, x^{*}\right)\right] .
$$

By virtue of the minimality of $I^{*}$, we know that $B^{-1} H\left(x^{*}, y^{*}\right)=\left(x^{*}, y^{*}\right)$ and so $H\left(x^{*}, y^{*}\right)=B\left(x^{*}, y^{*}\right)$. Therefore, $\left(x^{*}, y^{*}\right)$ is a minimal solution of the nonlinear operator equation $H(x, y)=B(x, y)$. It follows from Lemma 2.3 that $\left(x^{*}, y^{*}\right)$ is a coupled minimal-maximal solution of the nonlinear operator equation $N x=G(x, x)$. Now, Lemmas 2.1 and 2.2 imply that $\left(x^{*}, y^{*}\right)$ is a coupled minimal-maximal quasi-solution of the nonlinear operator equation (1.3). This completes the proof.

Remark 3.1. Theorems 3.1 and 3.2 presented in this paper generalize and improve Theorems 2.1 and 2.2 of Duan and Li [4], respectively. Therefore, our results also generalize and improve some corresponding results of Chen [3], Zhou and $\mathrm{Yu}$ [16], Liu and Wu [10], Syau [11], Zhang [14], Zhang and Xie [15].

Remark 3.2. We would like to point out Theorems 3.1 and 3.2 do not require any continuity for the operator $A$.

\section{References}

[1] S. S. Chang, Y. J. Cho, and H. Y. Zhou, Iterative Methods for Nonlinear Operator Equations in Banach Spaces, Nova Science Publishers, Inc., Huntington, NY, 2002.

[2] Y. Q. Chen and Y. J. Cho, Nonlinear Operator Theory in Abstract Spaces and Applications, Nova Science Publishers, Inc., New York, 2004.

[3] Y. Z. Chen, Fixed points of T-monotone operators, Nonlinear Anal. 24 (1995), no. 8, 1281-1287.

[4] H. G. Duan and G. Z. Li, The existence of couple minimal-maximal quasi-solutions for a class of nonlinear operator equations, J. Math. (Wuhan) 25 (2005), no. 5, 527-532.

[5] Y. Q. Feng and S. Y. Liu, Solvability of an operator equation in partial ordered space, Acta Math. Sinica (Chin. Ser.) 46 (2003), no. 2, 411-416.

[6] D. J. Guo and V. Lakshmikantham, Nonlinear Problems in Abstract Cone, Academic Press, New York, 1988.

[7] Coupled fixed points of nonlinear operators with applications, Nonlinear Anal. 11 (1987), no. 5, 623-632

[8] G. He, B. S. Lee, and N. J. Huang, Solvability of a new class of mixed monotone operator equations with an application, Nonlinear Anal. Forum 10 (2005), no. 2, 145-151.

[9] N. J. Huang, Y. Y. Tang, and Y. P. Liu, Some new existence theorems for nonlinear inclusion with an application, Nonlinear Funct. Anal. Appl. 6 (2001), no. 3, 341-350.

[10] X. Y. Liu and C. X. Wu, Fixed points of discontinuous weakly compact increasing operators and their applications to initial value problems in Banach spaces, J. Systems Sci. Math. Sci. 20 (2000), no. 2, 175-180.

[11] Y. Syau, Some fixed point theorems of T-monotone operators, J. Math. Anal. Appl. 205 (1997), no. 2, 325-329.

[12] S. Y. Xu and B. G. Jia, Fixed-point theorems of $\phi$ concave- $(-\psi)$ convex mixed monotone operators and applications, J. Math. Anal. Appl. 295 (2004), no. 2, 645-657.

[13] X. T. Yu, Geometric Theory in Banach Spaces, East China Normal University Press, Shanghai, 1986.

[14] K. M. Zhang, The solution of a nonlinear nonmonotone operator equation, Acta Math. Sci. (Chinese) 18 (1998), no. 2, 164-168. 
[15] K. M. Zhang and X. J. Xie, Solution and coupled minimal-maximal quasi-solutions of nonlinear non-monotone operator equations in Banach spaces, J. Math. Res. Exposition 23 (2003), no. 1, 47-52.

[16] Z. Zhou and Z. X. Yu, Fixed point theorems for mixed monotone operators and their applications, Gaoxiao Yingyong Shuxue Xuebao Ser. A 12 (1997), no. 3, 347-352.

YeOL Je CHO

Department of Mathematics Education and the RINS

Gyeongsang National University

Chinju 660-701, Korea

E-mail address: yjcho@gnu.ac.kr

GuANG He

Department of Mathematics

Neijiang Normal University

Neijiang, Sichuan 641112 , P. R. China

E-mail address: heguang6896@163.com

NAN-Jing Huang

Department of Mathematics

Sichuan University

Chengdu, Sichuan 610064, P. R. China

E-mail address: nanjinghuang@hotmail.com 